\title{
Sudden cardiac death in football players: Towards a new pre-participation algorithm (Review)
}

\author{
SOPHIE I. MAVROGENI ${ }^{1,2}$, KONSTANTINOS TSAROUHAS ${ }^{2,3}$, DEMETRIOS A. SPANDIDOS ${ }^{4}$ \\ CHRISTINA KANAKA-GANTENBEIN ${ }^{2,5}$ and FLORA BACOPOULOU ${ }^{2}$
}

\author{
${ }^{1}$ Onassis Cardiac Surgery Center, 17674 Athens; ${ }^{2}$ Exercise Physiology and Sports Medicine Clinic, \\ Center for Adolescent Medicine and UNESCO Chair on Adolescent Health Care, First Department of Pediatrics, \\ Medical School, National and Kapodistrian University of Athens, Aghia Sophia Children's Hospital, 11527 Athens; \\ ${ }^{3}$ Department of Cardiology, University Hospital of Larissa, 41110 Larissa; ${ }^{4}$ Department of Virology, \\ Medical School, University of Crete, 71003 Heraklion; ${ }^{5}$ First Department of Pediatrics, Medical School, \\ National and Kapodistrian University of Athens, Aghia Sophia Children's Hospital, 11527 Athens, Greece
}

Received September 19, 2018; Accepted November 22, 2018

DOI: 10.3892/etm.2018.7041

\begin{abstract}
Athletic pre-participation screening is essential for minimizing the risk for sudden cardiac death (SCD) in athletes participating in either competitive or leisure sporting activities. The primary causes of SCD in young athletes ( $<35$ years of age) include hypertrophic cardiomyopathy, congenital anomalies of the coronary artery and arrhythmogenic right ventricular cardiomyopathy. Other abnormalities, such as malignant arrhythmia due to blunt trauma to the chest (commotio cordis), myocarditis, valvular disease, aortic rupture (in Marfan syndrome) and ion channelopathies (catecholaminergic polymorphic ventricular tachycardia, Brugada syndrome, long or short QT syndrome), also contribute to a lesser degree to SCD. Currently, clinical assessment, electrocardiogram (ECG) and echocardiography are the cornerstones of the pre-participation athletic evaluation. However, their low
\end{abstract}

Correspondence to: Dr Flora Bacopoulou, Exercise Physiology and Sports Medicine Clinic, Center for Adolescent Medicine and UNESCO Chair on Adolescent Health Care, First Department of Pediatrics, Medical School, National and Kapodistrian University of Athens, Aghia Sophia Children's Hospital, 1 Thivon Street, 11527 Athens, Greece

E-mail: bacopouf@hotmail.com; fbacopoulou@med.uoa.gr

Abbreviations: CMR, cardiovascular magnetic resonance; SCD, sudden cardiac death; HCM, hypertrophic cardiomyopathy; ARVC, arrhythmogenic right ventricular cardiomyopathy; LVEF, left ventricular ejection fraction; ECG, electrocardiogram; LGE, late gadolinium enhancement; ECV, extracellular volume fraction; PPS, pre-participation screening; NSAIDs, non-steroidal anti-inflammatory drugs; COX-2, cyclooxygenase-2

Key words: football players, pre-participation examination, cardiomyopathy, coronary artery disease, myocardial inflammation, myocardial scar, symptoms, electrocardiogram, echocardiography, cardiovascular magnetic resonance sensitivity raises queries as regards the need for the application of more sophisticated modalities, such as cardiovascular magnetic resonance (CMR). CMR offers precise biventricular assessment and is greatly reproducible without the inherent limitations of echocardiography; i.e., low quality of images due to the lack of appropriate acoustic window or operator's experience. Furthermore, myocardium replacement fibrosis, indicative of patients' increased risk for future cardiac events, can be effectively detected by late gadolinium enhanced (LGE) images, acquired $15 \mathrm{~min}$ post-contrast injection. Finally, diffuse myocardial fibrosis not identified by LGE, can also be detected by pre-contrast (native) T1, post-contrast T1 mapping and extracellular volume images, which provide detailed information about the underlying pathophysiologic background. Therefore, CMR is recommended in all football players with a positive family or personal history of syncope or SCD, abnormal/doubtful ECG or echocardiogram.

\section{Contents}

1. Introduction

2. Physiology of cardiac stress in football

3. Causes of sudden cardiac death in football

4. Current guidelines for athletic PPS

5. Contribution of PPS to the prediction of SCD in football players

6. Echocardiography

7. Determining the added value of cardiovascular magnetic resonance imaging in predicting SCD in football players

8. The PPS algorithm proposed by our team

9. Conclusions

\section{Introduction}

Sudden cardiac death (SCD) is defined as any witnessed death occurring unexpectedly within $1 \mathrm{~h}$ of the onset of symptoms, 
or any unwitnessed death occurring within $24 \mathrm{~h}$ of having been seen alive and asymptomatic (1). Sport-related SCD is the death which occurs during or within $1 \mathrm{~h}$ post-exercise of moderate- to high-intensity (2).

Football (also known as soccer), with over 265 million registered players, attracts huge media attention and constitutes the most popular sport worldwide. SCD is not regarded as a frequent event in football; however the absolute numbers are not known. Examples of young famous players who suffered SCD are Miklós Fehér, Marc-Vivien Foe, Daniel Jarque and Antonio Puerta, who were considered healthy. Recreational football players, $>35$ years of age, with cardiovascular risk factors, are at risk of SCD.

Coronary artery disease (CAD) seems to be a leading cause of SCD in young football players ( $<35$ years of age). The potential pathophysiological mechanisms include increased catecholamine release and platelet aggregation, electrolyte abnormalities and dehydration. A retrospective study exclusively performed in football players in Turkey, revealed that CAD was the main cause of SCD, based on autopsies of this group (3). Furthermore, old infarctions and severe CAD have been found in very young (mean age, 25.7 years old) male athletes without cardiovascular risk factors, most of whom were recreational football players (4).

Diseases that are associated with SCD were identified in $0.38 \%$ of adolescent football players who underwent cardiovascular screening in the United Kingdom. The incidence of SCD was 6.8 per 100,000 athletes and the majority of these cases were due to cardiomyopathies, undetected on screening (5).

\section{Physiology of cardiac stress in football}

Football players have been selected due to their most advantageous anthropometric characteristics that enable them to compete at the greatest level of performance, which is a requirement for football (6). According to specific measurements, during a 90-min football match, players on the field run distances of approximately $10-12 \mathrm{~km}$ at the maximum level and the goalkeeper approximately $4 \mathrm{~km}$ (6). During the game, sprinting of 2-4 sec occurs approximately every $90 \mathrm{sec}$. A football match is a dynamic process during which tackling, heading and torso twisting to shift direction, and holding the ball against high defensive forces, are performed in addition to running. Although energy production during the football game depends mostly on aerobic metabolism, it is not uncommon to reach the anaerobic threshold; i.e., equal production and removal of lactate at the highest exercise level, approximately $80-90 \%$ of the maximal heart rate (6).

\section{Causes of SCD in football}

There is an age-dependent prevalence of the main pathologies that underlie SCD in athletes. The primary causes of SCD in young athletes ( $<35$ years of age) include hypertrophic cardiomyopathy (HCM), congenital anomalies of coronary artery and arrhythmogenic right ventricular cardiomyopathy (ARVC). Other abnormalities also contribute to SCD a lesser degree, and these include malignant arrhythmia due to blunt trauma to the chest (commotio cordis), myocarditis, valvular disease (aortic stenosis, mitral valve prolapse), aortic rupture (in Marfan syndrome) and ion channelopathies; i.e., catecholaminergic polymorphic ventricular tachycardia, Brugada syndrome and long or short QT syndrome (7-9). Therefore, Corrado et al, suggested that pre-participation screening (PPS) should be performed in all athletes participating in either competitive or leisure sporting activities (10).

Furthermore, CAD is the most common cause of SCD in older football players (>35 years of age) (10-12). Additionally, sickle cell trait (SCT) occurring in $8 \%$ of the African-American population, despite being considered a benign characteristic, has been related to the SCD of military recruits during vigorous physical activity. The 31-year United States Sudden Death in Athletes Registry was referred to as regards the deaths that occurred in competitive athletes in relation to SCT. Among the 2,462 deaths reported in athletes, 23 ( $0.9 \%$ of the whole population and $3.3 \%$ of African-Americans) were SCT-related; they were all African-Americans aged between 12 to 22 years, of whom $21(91 \%)$ were male. The majority of events occurred in the southern states with environmental temperatures $\geq 80^{\circ} \mathrm{F}$ during conditioning athletic training. Gradual deterioration over several minutes, associated with intense/exhaustive exercise and consequent collapse, were reported for each athlete (13).

In football players, doping has recently become an important matter, following the World Cup in 1994, when the superstar, Diego Maradona, after testing positive for forbidden substances, was expelled from the tournament (14). Androgen abuse has not only a direct effect on myocardial hypertrophy and fibrosis, but can also result in myocardial infarction, arrhythmia, hypertension and dyslipidemia (14-16). Herbal supplements, anti-histamines and non-steroidal anti-inflammatory drugs (NSAIDs), have also been implicated in SCD. Selective cyclooxygenase-2 (COX-2) NSAIDs for pain relief in arthritis or other musculoskeletal disorders, increase the risk of developing adverse cardiac outcomes (14). Second-generation anti-histamines (terfenadine and astemizole), through drug-food interactions, may reach high serum levels, predisposing to ventricular arrhythmias and prolonged QT (14).

The causes of athletic SCD differ among countries and from continent to continent. This is due to variations related to the geography and genetic background. HCM accounts for over a third of SCD cases in young competitive athletes in the United States (8) and ARVC represents $4 \%$ of SCD; nonetheless it is a major cause $(22 \%)$ of athletic SCD in Italy $(13,17)$. In the study by Corrado et al, the highest absolute number of sport-related SCDs was reported in football; however there was no significant association between the type of sports and SCD (18). The prevalence of premature CAD was surprisingly high in young athletes studied prospectively by Corrado et al (19). Suárez-Mier and Aguilera, analyzed athletic SCDs retrospectively, following a post-mortem examination of male recreational football players, aged (mean \pm SD) $24 \pm 8.8$ years. CAD was most frequently implicated in SCD and football was the second commonest sport related to SCD. A more detailed evaluation of the findings of that study, which is the only one analyzing SCD in relation to the type of sport, demonstrated that SCD in athletes occurs during or just after training or competi- 
tion (20). Even if the type of sport by itself is not responsible for the increased mortality during exertion, it can trigger SCD in those athletes with an underlying susceptibility (20).

\section{Current guidelines for athletic PPS}

Currently, there are no consensus guidelines regarding athletic PPS. The guidelines published (2004 and 2005) by the European Society of Cardiology and the International Olympic Committee are notably similar, but differ from the American guidelines (21) as they add a 12-lead electrocardiogram (ECG) to the assessment, beyond obtaining the athlete's history and performing a physical examination. This addition was mainly based on the significantly decreased incidence of SCD due to HCM in the Italian population, found in the study by Corrado et al (19). However, ECG as a screening method in the athletes' population has low sensitivity and specificity, which constitute a major limitation of its application (22).

In order to prevent football-related SCD, prior to the World Cup in 2006 in Germany, the Medical Assessment and Research Centre of the Fédération Internationale de Football Association (FIFA) developed and implemented a comprehensive pre-competition medical screening tool for this specific population (23). The cardiovascular assessment, apart from a thorough personal/family history and physical examination, also incorporated a 12-lead resting/exercise ECG and an echocardiogram. It has been demonstrated that cardiovascular PPS in international elite football teams is appropriate. While a more standardized ECG and echocardiogram could be useful, questions have arisen regarding exercise stress testing. Finally, although PPS was previously regarded as stressful for football players, due to the fear of being excluded, a study of professional football athletes in Norway found that PPS enhanced the players' confidence and they recommended it to other athletes (24).

\section{Contribution of PPS to the prediction of SCD in football players}

The PPS protocol developed by the European Society of Cardiology includes three components: i) Taking a thorough family and personal history; ii) performing a meticulous physical examination; and iii) conducting a 12-lead ECG. The ECG has $70 \%$ sensitivity in detecting the most common causes of SCD in young athletes $(7,8)$. In almost $1 / 3$ of these athletes who suffer an incipient cardiomyopathy, aortic disease or anomalous origin of coronary arteries, the ECG may be normal (25).

Intense somatic training induces structural and functional changes of the heart that present as altered 12-lead ECG. A total of 2,484 elite male football players from the French Professional Football League (2005-2015), underwent serial assessments with 12-lead ECGs. Of these, $17 \%$ presented sinus bradycardia ( $<50$ beats $/ \mathrm{min}), 8 \%$ demonstrated first-degree atrioventricular block and 3\% a prolonged QT interval, whereas $37 \%$ had left ventricular hypertrophy (LVH); i.e., a Sokolow-Lyon index (mean $\pm \mathrm{SD}$ ) of $34 \pm 10 \mathrm{~mm}$. Over time, a significant $(\mathrm{P}<0.001)$ remodeling with respect to decreased heart rate, QRS duration and QTcB delay was noted (26). In another study (27) evaluating players' health questionnaires along with 12-lead ECGs and echocardiograms, a significant percentage of athletes (9\%) was found to have clinically challenging ECG or structural abnormalities, which were difficult to differentiate from the physiologic adaptations of the 'athletic heart' or from potentially fatal states. Therefore, team physicians should be vigilant for the progressive cardiac risk of these individuals and ensure regular follow-up and attentive assessment (27).

\section{Echocardiography}

The low sensitivity of ECG in the PPS supports the implementation of a widely available, non-invasive modality such as the echocardiography. In the study by Rizzo et al, echocardiographic evaluations of 2,688 competitive athletes demonstrated abnormalities in 203 (7.5\%) of these. Athletic activity was prohibited in four athletes, two of which had $\mathrm{HCM}$, one had pulmonary valve stenosis and the other one had pectus excavatum compressing the right ventricle (RV). The other cardiac echocardiographic changes were insignificant (7.5\% of the total population) and therefore, only regular monitoring was recommended (28).

Various abnormalities can be detected in athletes' echocardiograms that could be characterized either as structural and functional cardiac adaptations that are within the physiological range and are typical to what is known the 'athlete's heart', or echocardiographic findings pointing to different cardiomyopathies that can induce SCD. The primary cause of SCD in young athletes is HCM. Using ECG as a diagnostic tool provides high sensitivity. Nevertheless, $10 \%$ of patients with HCM still present abnormal ECGs without the typical findings for hypertrophy. At the same time, $9 \%$ of the athletes with mild adaptive LVH, present ECGs with pathological changes (19). In both cases performing an echocardiogram is recommended in order to establish the appropriate diagnosis (29).

Half of the asymptomatic SCD cases in athletes are now attributed to the abnormal origin of the coronary arteries, despite the fact that in the past, such a condition was considered rare in SCD. In a large pool of asymptomatic children in the United States, anomalies of aortic origin was the second cause of sudden death associated with sports (30). In such athletes, the resting ECG is normal. Therefore, if the athlete is asymptomatic, this entity cannot be detected in a regular PPS based on personal history, physical examination with an ECG included. On the other hand, an adequately trained physician can differentiate coronary anomalies with high sensitivity using echocardiography (20). Therefore echocardiogram remains the key tool to uncover such asymptomatic patients (30).

Aortic root diseases are not a common SCD etiology in young individuals (19), although they are regarded as a more prevalent cause of SCD in athletes. Echocardiography allows both for the diagnosis and the follow-up of patients. Similarly, bicuspid aortic valve without significant functional abnormalities would not be diagnosed in a regular PPS, while again, echocardiography would allow for an early diagnosis and a proper follow-up (31).

Rest and stress echocardiography performed in 156 asymptomatic National Football League players in 
the United States, revealed that both wall thickness and left ventricular internal diameter (LVID) were increased and correlated with body size. The left ventricular ejection fraction (LVEF) was normal and no subject had an LVEF $<50 \%$, as expected. Regardless of the resting ejection fraction, all players presented hyperdynamic cardiac responses upon exercise (3). Echocardiographic indices have been successfully used to assess race differences in cardiac changes occurring in football players. Black players were characterized by higher LV mass indices (i.e., higher mass/volume ratios and higher QRS vector magnitudes) and exhibited more concentric ventricular remodeling, lower early diastolic annular velocities and increased ventricular voltage, compared to their Caucasian counterparts. On the other hand, ventricular mass increased proportionally to volume in white players but not in black players (32).

\section{Determining the added value of cardiovascular magnetic resonance imaging in predicting SCD in football players}

It is evident that the currently used non-invasive approach including clinical evaluation and ECG and occasionally echocardiographic evaluation cannot entirely exclude the risk of SCD. Therefore, a more detailed approach should be recommended, at least in those football players with high clinical suspicion. Cardiovascular magnetic resonance (CMR) offers an excellent non-invasive and radiation-free approach in order to characterize cardiac anatomy, physiology and cardiac remodeling, which are prerequisites for assessing athletes (33). CMR provides three-dimensional tomographic imaging with high spatial and temporal resolution and cine imaging sequences (i.e., steady-state free precession). In this manner, clear delineation of the endocardial and epicardial borders can be achieved, along with precise wall thickness measurements at any point of the LV. Furthermore, CMR provides tomographic imaging by acquiring a stack of short-axis images, with full ventricular coverage offering precise biventricular assessment. In addition, great vessels can be monitored along with the entire LV-RV for abnormalities, including anomalous origin of the coronary arteries, focal hypertrophy, regional wall motion changes, myocardial inflammation and/or fibrosis (33).

CMR images share high reproducibility without the inherent limitations of echocardiography, such as low image quality due to lack of appropriate acoustic window or the operator's skills (34). Most importantly, as breaking data have indicated, patients at an increased risk of developing future cardiac events can be identified by the presence of replacement myocardial fibrosis, visible in late gadolinium enhanced (LGE) images, taken 15 min post contrast-enhanced CMR sequences (35). Moreover, through the evaluation of pre-contrast (native) T1, post-contrast T1 mapping and extracellular volume (ECV) images, diffuse myocardial fibrosis, which is undetectable by LGE can be identified and detailed information of the underlying pathophysiological background can be provided (36).

\section{The PPS algorithm proposed by our team}

All football players either professional or recreational should undergo thorough clinical, ECG and echocardiographic evaluation. Those with positive family or personal history of syncope or SCD, abnormal/doubtful ECG or echocardiogram, should be also evaluated by CMR. The CMR imaging should include assessment of the following: i) Origin and coronary arteries abnormalities; ii) right-left ventricular function; iii) adenosine stress perfusion and replacement fibrosis (LGE); iv) native T1 and T2 mapping for edema; and v) post-contrast T1 mapping and ECV for diffuse fibrosis. The above-described protocol can clarify all doubtful cases and potentially eliminate the risk of SCD among football players.

\section{Conclusions}

The international literature describes HCM, ARVC and congenital coronary artery anomalies as the leading etiology of SCD in athletes who are $<35$ years of age. Currently, clinical evaluation, ECG and echocardiography are the cornerstones of the athletic pre-participation evaluation. However, their low sensitivity raises queries about the necessity for application of more sophisticated modalities, such as CMR that offers precise, highly reproducible biventricular assessment without the inherent limitations of echocardiography. Most importantly, patients at an increased risk of developing future cardiac events can be identified by the presence of replacement myocardial fibrosis, visible in LGE images, taken $15 \mathrm{~min}$ post-contrastinjection. Furthermore, the evaluation of pre-contrast (native) T1, post-contrast T1 mapping and ECV images can identify diffuse myocardial fibrosis, undetectable by LGE and provide detailed information about the underlying pathophysiological background. Therefore, it is recommended for all football players with positive family or personal history of syncope or SCD, abnormal/doubtful ECG or echocardiogram.

\section{Acknowledgements}

The authors are responsible for the choice and presentation of views contained in this article and for opinions expressed therein, which are not necessarily those of UNESCO and do not commit the Organization.

\section{Funding}

No funding was received.

\section{Availability of data and materials}

Not applicable.

\section{Authors' contributions}

SIM, KT and FB recognized the scientific need for a review article on this topic and conceived the study. SIM and DAS designed the study structure. SIM and CK-G performed the literature search and screened the findings. KT and FB independently reviewed the literature search. SIM drafted the manuscript. FB and KT reviewed the original draft. DAS and CK-G substantially reviewed and enriched the manuscript and performed the guidelines cross-check. All authors have read and approved the final manuscript. 


\section{Ethics approval and consent to participate}

Not applicable.

\section{Patient consent for publication}

Not applicable.

\section{Competing interests}

DAS is the Editor-in-Chief for the journal, but had no personal involvement in the reviewing process, or any influence in terms of adjudicating on the final decision, for this article.

\section{References}

1. Davogustto G and Higgins J: Sudden cardiac death in the soccer field: A retrospective study in young soccer players from 2000 to 2013. Phys Sportsmed 42: 20-29, 2014.

2. Chugh SS, Jui J, Gunson K, Stecker EC, John BT, Thompson B Ilias N, Vickers C, Dogra V, Daya M, et al: Current burden of sudden cardiac death: Multiple source surveillance versus retrospective death certificate-based review in a large U.S community. J Am Coll Cardiol 44: 1268-1275, 2004.

3. Abernethy WB III, Choo JK and Hutter AM Jr: Echocardiographic characteristics of professional football players. J Am Coll Cardiol 41: 280-284, 2003

4. Holst AG, Winkel BG, Theilade J, Kristensen IB, Thomsen JL, Ottesen GL, Svendsen JH, Hauns $\varnothing$ S, Prescott E and Tfelt-Hansen J: Incidence and etiology of sports-related sudden cardiac death in Denmark--implications for preparticipation screening. Heart Rhythm 7: 1365-1371, 2010.

5. Malhotra A, Dhutia H, Finocchiaro G, Gati S, Beasley I, Clift P, Cowie C, Kenny A, Mayet J, Oxborough D, et al: Outcomes of Cardiac Screening in Adolescent Soccer Players. N Engl J Med 379: 524-534, 2018.

6. Stølen T, Chamari K, Castagna C and Wisløff U: Physiology of soccer: An update. Sports Med 35: 501-536, 2005.

7. Ozdemir C, Saka T, Asil H, Uzün I and Oner M: Soccer related sudden deaths in Turkey. J Sports Sci Med 7: 292-298, 2008.

8. Borjesson M and Pelliccia A: Incidence and aetiology of sudden cardiac death in young athletes: An international perspective. $\mathrm{Br}$ J Sports Med 43: 644-648, 2009.

9. Maron BJ: Sudden death in young athletes. N Engl J Med 349: 1064-1075, 2003.

10. Corrado D, Schmied C, Basso C, Borjesson M, Schiavon M, Pelliccia A, Vanhees L and Thiene G: Risk of sports: Do we need a pre-participation screening for competitive and leisure athletes? Eur Heart J 32: 934-944, 2011.

11. Meyer L, Stubbs B, Fahrenbruch C, Maeda C, Harmon K, Eisenberg $\mathrm{M}$ and Drezner J: Incidence, causes, and survival trends from cardiovascular-related sudden cardiac arrest in children and young adults 0 to 35 years of age: A 30-year review. Circulation 126: 1363-1372, 2012.

12. Stamatopoulos PG, Dangas G, Tsarouhas K, Ziogas G, Tsitsimpikou C, Stamatopoulos G and Chrousos G: Subtotal Occlusion of Left Anterior Coronary Artery in a Professional Athlete. Cardiology 140: 71-73, 2018.

13. Harmon KG, Drezner JA, Klossner D and Asif IM: Sickle cell trait associated with a RR of death of 37 times in National Collegiate Athletic Association football athletes: A database with 2 million athlete-years as the denominator. Br J Sports Med 46: 325-330, 2012

14. Higgins JP and Andino A: Soccer and Sudden Cardiac Death in Young Competitive Athletes: A Review. J Sports Med (Hindawi Publ Corp): March 26, 2013 (Epub ahead of print). doi: $10.1155 / 2013 / 967183$.

15. Germanakis I, Tsarouhas K, Fragkiadaki P, Tsitsimpikou C, Goutzourelas N, Champsas MC, Stagos D, Rentoukas E and Tsatsakis AM: Oxidative stress and myocardial dysfunction in young rabbits after short term anabolic steroids administration. Food Chem Toxicol 61: 101-105, 2013.
16. Vasilaki F, Tsitsimpikou C, Tsarouhas K, Germanakis I, Tzardi M, Kavvalakis M, Ozcagli E, Kouretas D and Tsatsakis AM: Cardiotoxicity in rabbits after long-term nandrolone decanoate administration. Toxicol Lett 241: 143-151, 2016.

17. Maron BJ, Thompson PD, Ackerman MJ, Balady G, Berger S, Cohen D, Dimeff R, Douglas PS, Glover DW, Hutter AM Jr, et al; American Heart Association Council on Nutrition, Physical Activity, and Metabolism: Recommendations and considerations related to preparticipation screening for cardiovascular abnormalities in competitive athletes: 2007 update: a scientific statement from the American Heart Association Council on Nutrition, Physical Activity, and Metabolism: endorsed by the American College of Cardiology Foundation. Circulation 115: 1643-455, 2007.

18. Corrado D, Basso C, Schiavon M and Thiene G: Screening for hypertrophic cardiomyopathy in young athletes. N Engl J Med 339: 364-369, 1998.

19. Corrado D, Basso C, Rizzoli G, Schiavon M and Thiene G: Does sports activity enhance the risk of sudden death in adolescents and young adults? J Am Coll Cardiol 42: 1959-1963, 2003.

20. Suárez-Mier MP and Aguilera B: [Causes of sudden death during sports activities in Spain]. Rev Esp Cardiol 55: 347-358, 2002 (In Spanish)

21. Bille K, Figueiras D, Schamasch P, Kappenberger L, Brenner JI, Meijboom FJ and Meijboom EJ: Sudden cardiac death in athletes: The Lausanne Recommendations. Eur J Cardiovasc Prev Rehabil: 13: 859-875, 2006.

22. Pelliccia A, Di Paolo FM, Quattrini FM, Basso C, Culasso F, Popoli G, De Luca R, Spataro A, Biffi A, Thiene $\mathrm{G}$ and Maron BJ: Outcomes in athletes with marked ECG repolarization abnormalities. N Engl J Med 358: 152-161, 2008.

23. Thünenkötter T, Schmied C, Dvorak J and Kindermann W: Benefits and limitations of cardiovascular pre-competition screening in international football. Clin Res Cardiol 99: 29-35, 2010.

24. Solberg EE, Bjørnstad TH, Andersen TE and Ekeberg Ø: Cardiovascular pre-participation screening does not distress professional football players. Eur J Prev Cardiol 19: 571-577, 2012.

25. Corrado D, Pelliccia A, Bjørnstad HH, Vanhees L, Biffi A, Borjesson M, Panhuyzen-Goedkoop N, Deligiannis A, Solberg E, Dugmore D, et al; Study Group of Sport Cardiology of the Working Group of Cardiac Rehabilitation and Exercise Physiology and the Working Group of Myocardial and Pericardial Diseases of the European Society of Cardiology; Consensus Statement of the Study Group of Sport Cardiology of the Working Group of Cardiac Rehabilitation and Exercise Physiology and the Working Group of Myocardial and Pericardial Diseases of the European Society of Cardiology: Cardiovascular pre-participation screening of young competitive athletes for prevention of sudden death: Proposal for a common European protocol. Eur Heart J 26: 516-524, 2005.

26. Huttin O, Selton-Suty C, Venner C, Vilain JB, Rochecongar P and Aliot E: Electrocardiographic patterns and long-term training-induced time changes in 2484 elite football players. Arch Cardiovasc Dis 111: 380-388, 2018.

27. Speers C, Seth A, Patel K, Gillett M and Rakhit D: The heart of the matter: Cardiac assessment in professional footballers. Br J Sports Med: doi: 10.1136/bjsports-2017-097827.2.

28. Rizzo M, Spataro A, Cecchetelli C, Quaranta F, Livrieri S, Sperandii F, Cifra B, Borrione P and Pigozzi F: Structural cardiac disease diagnosed by echocardiography in asymptomatic young male soccer players: Implications for pre-participation screening. Br J Sports Med 46: 371-373, 2012

29. Rowin EJ, Maron BJ, Appelbaum E, Link MS, Gibson CM, Lesser JR, Haas TS, Udelson JE, Manning WJ and Maron MS: Significance of false negative electrocardiograms in preparticipation screening of athletes for hypertrophic cardiomyopathy. Am J Cardiol 110: 1027-1032, 2012.

30. Lytrivi ID, Wong AH, Ko HH, Chandra S, Nielsen JC, Srivastava S, Lai WW and Parness IA: Echocardiographic diagnosis of clinically silent congenital coronary artery anomalies. Int J Cardiol 126: 386-393, 2008.

31. Stefani L, De Luca A, Maffulli N, Mercuri R, Innocenti G, Suliman I, Toncelli L, Vono MC, Cappelli B, Pedri $\mathrm{S}$, et al: Speckle tracking for left ventricle performance in young athletes with bicuspid aortic valve and mild aortic regurgitation. Eur J Echocardiogr 10: 527-531, 2009. doi: 10.1093/ejechocard/jen332. 
32. Haddad F, Peter S, Hulme O, Liang D, Schnittger I, Puryear J, Gomari FA, Finocchiaro G, Myers J, Froelicher V, et al: Race differences in ventricular remodeling and function among college football players. Am J Cardiol 112: 128-134, 2013.

33. Hundley WG, Bluemke DA, Finn JP, Flamm SD, Fogel MA, Friedrich MG, Ho VB, Jerosch-Herold M, Kramer CM, Manning WJ, et al; American College of Cardiology Foundation Task Force on Expert Consensus Documents: ACCF/ACR/AHA/NASCI/SCMR 2010 expert consensus document on cardiovascular magnetic resonance: A report of the American College of Cardiology Foundation Task Force on Expert Consensus Documents. Circulation 121: 2462-2508, 2010.

34. Maron MS: Clinical utility of cardiovascular magnetic resonance in hypertrophic cardiomyopathy. J Cardiovasc Magn Res 14: 13, 2012. doi: 10.1186/1532-429X-14-13.
35. Gulati A, Jabbour A, Ismail TF, Guha K, Khwaja J, Raza S, Morarji K, Brown TD, Ismail NA, Dweck MR, et al: Association of fibrosis with mortality and sudden cardiac death in patients with nonischemic dilated cardiomyopathy. JAMA 309: 896-908, 2013.

36. Messroghli DR, Moon JC, Ferreira VM, et al.: Clinical recommendations for cardiovascular magnetic resonance mapping of T1, T2, T2* and extracellular volume: A consensus statement by the Society for Cardiovascular Magnetic Resonance (SCMR) endorsed by the European Association for Cardiovascular Imaging (EACVI). J Cardiovasc Magn Reson: 19: 75, 2017.

This work is licensed under a Creative Commons Attribution-NonCommercial-NoDerivatives 4.0 International (CC BY-NC-ND 4.0) License. 